This is a preprint of: "Limit cycles bifurcating from a family of reversible quadratic centers via averaging theory", Jaume Llibre, Arefeh Nabavi, Marzieh Mousavi, Internat. J. Bifur. Chaos Appl. Sci. Engrg., vol. 30(4), 2050051:1-8, 2020.

DOI: [10.1142/S0218127420500510]

\title{
LIMIT CYCLES BIFURCATING FROM A FAMILY OF REVERSIBLE QUADRATIC CENTERS VIA AVERAGING THEORY
}

\author{
JAUME LLIBRE ${ }^{1}$, AREFEH NABAVI ${ }^{2}$ AND MARZIEH MOUSAVI ${ }^{2}$
}

ABStract. Consider the class of reversible quadratic systems

$$
\dot{x}=y, \quad \dot{y}=-x+x^{2}+y^{2}-r^{2},
$$

with $r>0$. These quadratic polynomial differential systems have a center at the point $\left(\left(1-\sqrt{1+4 r^{2}}\right) / 2,0\right)$ and the circle $x^{2}+y^{2}=r^{2}$ is one of the periodic orbits surrounding this center. These systems can be written into the form

$$
\dot{x}=y+(4+A) x^{2}-A y^{2}, \quad \dot{y}=-x,
$$

with $A \in(-2,0)$. We prove for all $A \in \mathbb{R}$ that the averaging theory up to seven order applied to this last system perturbed inside the whole class of quadratic polynomial differential systems can produce at most two limit cycles (respectively one limit cycle) bifurcating from the periodic orbits surrounding the center $(0,0)$ of that system. Up to now this result was only know for $A=-2$ (see $[22,23])$.

\section{IntRoduction AND STATEMENT OF THE MAIN RESULTS}

One of the important problems in the qualitative theory of differential equations is the study of their limit cycles. The second part of the well-known Hilbert's 16th problem [26] asks about the maximal number and the possible relative positions of limit cycles in the planar polynomial differential systems of degree $n \geq 2$. This problem is still open, even for the case $n=2$.

An easier problem than the Hilbert's 16th problem is the study of the number of limit cycles which can bifurcate from the periodic orbits surrounding a center of a polynomial differential system. Many authors these last years have studied this last problem restricted to the centers of the quadratic polynomial differential systems, see for instance the

2010 Mathematics Subject Classification. Primary 34A05. Secondary 34C05, $37 \mathrm{C} 10$.

Key words and phrases. limit cycles, quadratic reversible centers, averaging theory. 


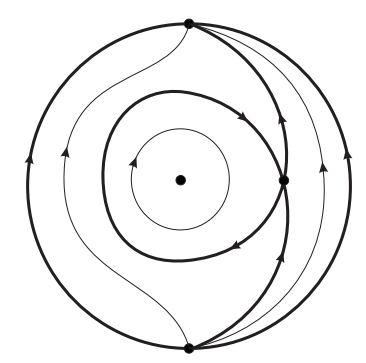

Figure 1. The phase portrait in the Poincaré disc of system (1).

book of Christopher and $\mathrm{Li}[6]$ and the hundreds of references quoted therein.

The tools for studying the limit cycles bifurcating from the periodic orbits surrounding a center are the Poincaré return map (see for instance $[3,20]$ ), the Poincaré-Melnikov integrals (see for example $[13,14]$ ), the Abelian integrals (see $[1,6,28]$ ), the averaging theory (see for instance $[2,7]$ ), and the inverse integrating factor (see [11]). While the first three methods only provide the number of bifurcated limit cycles, the averaging method and method which use the inverse integrating factor can also give the shape of bifurcated limit cycles. For a general overview and more details about the mentioned methods see reference [12].

According with Iliev [17] and using complex notation the centers of the quadratic polynomial differential systems are classified into the following five families:

$\dot{z}=-i z-z^{2}+2|z|^{2}+(\bar{b}+i \bar{c}) \bar{z}^{2}, \quad$ Hamiltonian $\left(Q_{3}^{H}\right)$,

$\dot{z}=-i z+a z^{2}+2|z|^{2}+b \bar{z}^{2}, \quad$ Reversible $\left(Q_{3}^{R}\right)$,

$\dot{z}=-i z+4 z^{2}+2|z|^{2}+(b+i c) \bar{z}^{2}, \quad|b+i c|=2$, codimension four $\left(Q_{4}\right)$,

$\dot{z}=-i z+z^{2}+(b+i c) \bar{z}^{2}, \quad$ Generalized Lotka-Voltera $\left(Q_{3}^{L V}\right)$,

$\dot{z}=-i z+\bar{z}^{2}, \quad$ Hamiltonian triangle,

where $a, b, c$ are arbitrary real constants.

The major part of papers studying the bifurcation of limit cycles from the periodic orbits surrounding a center consider centers of polynomial Hamiltonian systems. Thus this problem for the centers of the quadratic polynomial Hamiltonian systems has been solved completely, see $[5,10,15,16,19,21]$. 
Here for $r>0$ we consider the following family of quadratic polynomial differential systems

$$
\dot{x}=y, \quad \dot{y}=-x+x^{2}+y^{2}-r^{2},
$$

which have the circle $x^{2}+y^{2}=r^{2}$ as a periodic orbit surrounding the center $\left(\left(1-\sqrt{1+4 r^{2}}\right) / 2,0\right)$. The phase portraits in the Poincaré disc of these quadratic polynomial differential systems have been studied in [25], see Figure 1. It is easy to check that system (1) has the nonalgebraic first integral

$$
H(x, y)=e^{-2 x}\left(x^{2}+y^{2}-r^{2}\right) .
$$

Taking $z=x+i y$ we obtain that any quadratic reversible center $Q_{3}^{R}$ can be written in the following real form

(2) $\dot{x}=y+(a+b+2) x^{2}-(a+b-2) y^{2}, \quad \dot{y}=-x[1-2(a-b) y]$.

There are some papers studying the limit cycles which bifurcate from the periodic orbits surrounding the centers of $(2)$ with $a \neq b$, see for instance $[4,18]$. The study of the case $a=b$ is more difficult because the first integral contains an exponential function. The following lemma shows that system (1) is a reversible system (2) with $a=b$.

Lemma 1. Systems (1) are inside the class of $Q_{3}^{R}$ because they can be written as

$$
\dot{x}=y+(4+A) x^{2}-A y^{2}, \quad \dot{y}=-x,
$$

where $a=b=1+\frac{A}{2}$ and $A \in(-2,0)$.

This lemma is proved in section 3 .

As far as we know the first work on the bifurcation of limit cycles from the periodic orbits surrounding a center of a system (3) under quadratic perturbations was done by $\mathrm{Li}[22]$ for $A=-2$ proving that at most 2 limit cycles bifurcate from that center. Note that for $A=-2$ system (3) with the change of variables $(x, y, t) \rightarrow(x / 2, y / 2,-t)$ becomes the system

$$
\dot{x}=-y-x^{2}-y^{2}, \quad \dot{y}=x,
$$

studied by Li. Later on Liu [23] provided a shorter proof of the result of Li. In this paper we study this problem for all values of $A$.

From statement (ii) of Theorem 1 in Iliev [17] the essential quadratic perturbations for finding the upper bound on the number of limit cycles which can bifurcate from the periodic orbits surrounding the center 
$(0,0)$ of the reversible quadratic polynomial differential systems (3) under general quadratic perturbations are

$$
\dot{z}=\left(\lambda_{1}(\varepsilon)-i\right) z+\left(a+i \lambda_{3}(\varepsilon)\right) z^{2}+2|z|^{2}+\left(b+i \lambda_{5}(\varepsilon)\right) \bar{z}^{2},
$$

where we can take the $\lambda_{k}(\varepsilon)$ for $k=1,3,5$ as real analytical functions in $\varepsilon$ sufficiently small with $\lambda_{k}(0)=0$. That is $\lambda_{k}(\varepsilon)=\sum_{j=1}^{\infty} \lambda_{k j} \varepsilon^{j}$. In fact according with Iliev it would be sufficient to take $\lambda_{k}(\varepsilon)=\lambda_{k} \varepsilon$, but since we shall use averaging theory up to order seven for studying the limit cycles of system (4) it is convenient to take $\lambda_{k}(\varepsilon)=\sum_{j=1}^{\infty} \lambda_{k j} \varepsilon^{j}$.

In short, for studying the maximum number of limit cycles which can bifurcate from the periodic orbits surrounding the center $(0,0)$ of system (3) perturbed by a general quadratic polynomial, it is enough to study for $\varepsilon \neq 0$ sufficiently small the limit cycles of system (4), or equivalently the limit cycles of system (4) written in the real variables $(x, y)$ where $z=x+i y$, i.e.

$$
\begin{aligned}
& \dot{x}=y+(4+A) x^{2}-A y^{2}+\sum_{i=1}^{\infty} \varepsilon^{i}\left(\lambda_{1 i} x+2\left(\lambda_{5 i}-\lambda_{3 i}\right) x y\right), \\
& \dot{y}=-x+\sum_{i=1}^{\infty} \varepsilon^{i}\left(\lambda_{1 i} y+\left(\lambda_{3 i}+\lambda_{5 i}\right)\left(x^{2}-y^{2}\right)\right) .
\end{aligned}
$$

Our main result is the following one.

Theorem 2. For $|\varepsilon| \neq 0$ sufficiently small and for all $A \in \mathbb{R}$ the maximum number of limit cycles of system (5) obtained using averaging theory up to seven order is two.

Theorem 2 is proved in section 3 .

As far as we know in this paper it is the first time that it is used in an application the average functions until order seven. We note, as it is shown in the proof of Theorem 2, that the two limit cycles can be obtained already from the fifth average function when $A \in \mathbb{R} \backslash\{-4,6\}$, and only from the seventh average function when $A \in\{-4,6\}$.

\section{Preliminary Results}

In this section we recall the results on the averaging theory that we need for proving Theorem 2 .

We consider a differential system of the form

$$
\dot{x}=\sum_{i=1}^{k} \varepsilon^{i} F_{i}(t, x)+\varepsilon^{k+1} G(t, x, \varepsilon),
$$


where $F_{i}: \mathbb{R} \times D \rightarrow \mathbb{R}^{n}$ for $i=1, \ldots, k$, and $G: \mathbb{R} \times D \times\left(-\varepsilon_{0}, \varepsilon_{0}\right) \rightarrow \mathbb{R}^{n}$ are continuous functions and T-periodic in the first variable, $D$ is an open subset of $\mathbb{R}^{n}$ and $\varepsilon$ a small parameter.

From [27] (see also (1.103), (1.104) and section 1.3.4 of [24]) we define the functions $y_{i}(t, z)$ and the average functions $f_{i}(z)$ for $i=1, \ldots, 7$ associated to system (6). In these functions we denote by $\partial^{k} F_{m}(s, z)$ the $k$-th partial derivative of the function $F_{m}(s, z)$ with respect to the variable $z$. Thus we have

$$
\begin{aligned}
& y_{1}(t, z)=\int_{0}^{t} F_{1}(s, z) d s \\
& y_{2}(t, z)=\int_{0}^{t}\left(2 F_{2}(s, z)+2 \partial F_{1}(s, z) y_{1}(s, z)\right) d s, \\
& y_{3}(t, z)=\int_{0}^{t}\left(6 F_{3}(s, z)+6 \partial F_{2}(s, z) y_{1}(s, z)+3 \partial^{2} F_{1}(s, z) y_{1}(s, z)^{2}\right. \\
& \left.+3 \partial F_{1}(s, z) y_{2}(s, z)\right) d s, \\
& y_{4}(t, z)=\int_{0}^{t}\left(24 F_{4}(s, z)+24 \partial F_{3}(s, z) y_{1}(s, z)+12 \partial^{2} F_{2}(s, z) y_{1}(s, z)^{2}\right. \\
& +12 \partial F_{2}(s, z) y_{2}(s, z)+12 \partial^{2} F_{1}(s, z) y_{1}(s, z) y_{2}(s, z) \\
& \left.+4 \partial^{3} F_{1}(s, z) y_{1}(s, z)^{3}+4 \partial F_{1}(s, z) y_{3}(s, z)\right) d s, \\
& y_{5}(t, z)=\int_{0}^{t}\left(120 F_{5}(s, z)+120 \partial F_{4}(s, z) y_{1}(s, z)+60 \partial^{2} F_{3}(s, z) y_{1}(s, z)^{2}\right. \\
& +60 \partial F_{3}(s, z) y_{2}(s, z)+60 \partial^{2} F_{2}(s, z) y_{1}(s, z) y_{2}(s, z) \\
& \left.+20 \partial^{3} F_{2}(s, z) y_{1}(s, z)^{3}+20 \partial F_{2}(s, z) y_{3}(s, z)\right) \\
& +20 \partial^{2} F_{1}(s, z) y_{1}(s, z) y_{3}(s, z)+15 \partial^{2} F_{1}(s, z) y_{2}(s, z)^{2} \\
& +30 F_{1}(s, z) y_{1}(s, z)^{2} y_{2}(s, z)+5 \partial^{4} F_{1}(s, z) y_{1}(s, z)^{4} \\
& \left.+5 \partial F_{1}(s, z) y_{4}(s, z)\right) d s \text {. } \\
& y_{6}(t, z)=\int_{0}^{t}\left(720 F_{6}(s, z)+720 \partial F_{5}(s, z) y_{1}(s, z)+360 \partial F_{4}(s, z) y_{2}(s, z)\right. \\
& +360 \partial^{2} F_{4}(s, z) y_{1}(s, z)^{2}+120 \partial F_{3}(s, z) y_{3}(s, z) \\
& +360 \partial^{2} F_{3}(s, z) y_{1}(s, z) y_{2}(s, z)+120 \partial^{3} F_{3}(s, z) y_{1}(s, z)^{3} \\
& +30 \partial F_{2}(s, z) y_{4}(s, z)+120 \partial^{2} F_{2}(s, z) y_{1}(s, z) y_{3}(s, z) \\
& +30 \partial^{4} F_{2}(s, z) y_{1}(s, z)^{4}+90 \partial^{2} F_{2}(s, z) y_{2}(s, z)^{2} \\
& +180 \partial^{3} F_{2} y_{1}(s, z)^{2} y_{2}(s, z)+6 \partial F_{1}(s, z) y_{5}(s, z)
\end{aligned}
$$




$$
\begin{aligned}
& +30 \partial^{2} F_{1}(s, z) y_{1}(s, z) y_{4}(s, z)+60 \partial^{2} F_{1}(s, z) y_{2}(s, z) y_{3}(s, z) \\
& +60 \partial^{3} F_{1}(s, z) y_{1}(s, z)^{2} y_{3}(s, z)+60 \partial^{4} F_{1}(s, z) y_{1}(s, z)^{3} y_{2}(s, z) \\
& \left.+90 \partial^{3} F_{1}(s, z) y_{1}(s, z) y_{2}(s, z)^{2}+6 \partial^{5} F_{1}(s, z) y_{1}(s, z)^{5}\right) d s
\end{aligned}
$$

Moreover we have

$$
\begin{aligned}
& f_{1}(z)= \frac{1}{T} \int_{0}^{T} F_{1}(t, z) d t \\
& f_{2}(z)= \frac{1}{T} \int_{0}^{T}\left(F_{2}(t, z)+\partial F_{1}(t, z) y_{1}(t, z)\right) d t \\
& f_{3}(z)= \frac{1}{T} \int_{0}^{T}\left(F_{3}(t, z)+\partial F_{2}(t, z) y_{1}(t, z)+\frac{1}{2} \partial^{2} F_{1}(t, z) y_{1}(t, z)^{2}+\right. \\
&\left.+\frac{1}{2} \partial F_{1}(t, z) y_{2}(t, z)\right) d t \\
& f_{4}(z)= \frac{1}{T} \int_{0}^{T}\left(F_{4}(t, z)+\partial F_{3}(t, z) y_{1}(t, z)+\frac{1}{2} \partial^{2} F_{2}(t, z) y_{1}(t, z)^{2}\right. \\
&+\frac{1}{2} \partial F_{2}(t, z) y_{2}(t, z)+\frac{1}{2} \partial^{2} F_{1}(t, z) y_{1}(t, z) y_{2}(t, z) \\
&\left.+\frac{1}{6} \partial^{3} F_{1}(t, z) y_{1}(t, z)^{3}+\frac{1}{6} \partial F_{1}(t, z) y_{3}(t, z)\right) d t \\
& \frac{1}{T} \int_{0}^{T}\left(F_{5}(t, z)+\partial F_{4}(t, z) y_{1}(t, z)+\frac{1}{2} \partial^{2} F_{3}(t, z) y_{1}(t, z)^{2}\right. \\
&+\frac{1}{2} \partial F_{3}(t, z) y_{2}(t, z)+\frac{1}{2} \partial^{2} F_{2}(t, z) y_{1}(t, z) y_{2}(t, z) \\
&+\frac{1}{6} \partial^{3} F_{2}(t, z) y_{1}(t, z)^{3}+\frac{1}{6} \partial F_{2}(t, z) y_{3}(t, z) \\
&+\frac{1}{6} \partial^{2} F_{1}(t, z) y_{1}(t, z) y_{3}(t, z)+\frac{1}{8} \partial^{2} F_{1}(t, z) y_{2}(t, z)^{2} \\
&+\frac{1}{4} \partial^{3} F_{1}(t, z) y_{1}(t, z)^{2} y_{2}(t, z)+\frac{1}{24} \partial^{4} F_{1}(t, z) y_{1}(t, z)^{4} \\
&\left.+\frac{1}{24} \partial F_{1}(t, z) y_{4}(t, z)\right) d s . \\
& \frac{1}{T} \int_{0}^{T}\left(F_{6}(t, z)+\partial F_{5}(t, z) y_{1}(t, z)+\frac{1}{2} \partial F_{4}(t, z) y_{2}(t, z)\right. \\
&+\frac{1}{2} \partial^{2} F_{4}(t, z) y_{1}(t, z)^{2}+\frac{1}{6} \partial F_{3}(t, z) y_{3}(t, z) \\
&+\frac{1}{2} \partial^{2} F_{3}(t, z) y_{1}(t, z) y_{2}(t, z)+\frac{1}{6} \partial^{3} F_{3}(t, z) y_{1}(t, z)^{3} \\
&+\frac{1}{24} \partial F_{2}(t, z) y_{4}(t, z)+\frac{1}{6} \partial^{2} F_{2}(t, z) y_{1}(t, z) y_{3}(t, z) \\
& f_{6}(z) \partial^{3} F_{2}(t, z) y_{1}(t, z)^{2} y_{2}(t, z)+\frac{1}{8} \partial^{2} F_{2}(t, z) y_{2}(t, z)^{2} \\
&\left.f_{5}\right)
\end{aligned}
$$




$$
\begin{aligned}
& +\frac{1}{24} \partial^{4} F_{2}(t, z) y_{1}(t, z)^{4}+\frac{1}{120} \partial F_{1}(t, z) y_{5}(t, z) \\
& +\frac{1}{24} \partial^{2} F_{1}(t, z) y_{1}(t, z) y_{4}(t, z)+\frac{1}{12} \partial^{2} F_{1}(t, z) y_{2}(t, z) y_{3}(t, z) \\
& +\frac{1}{12} \partial^{3} F_{1}(t, z) y_{1}(t, z)^{2} y_{3}(t, z)+\frac{1}{12} \partial^{4} F_{1}(t, z) y_{1}(t, z)^{3} y_{2}(t, z) \\
& \left.+\frac{1}{8} \partial^{3} F_{1}(t, z) y_{1}(t, z) y_{2}(t, z)^{2}+\frac{1}{120} \partial^{5} F_{1}(t, z) y_{1}(t, z)^{5}\right) d t \text {. } \\
& f_{7}(z)=\frac{1}{T} \int_{0}^{T}\left(F_{7}(s, z)+\partial F_{6}(s, z) y_{1}(s, z)\right. \\
& +\frac{1}{2} \partial F_{5}(s, z) y_{2}(s, z)+\frac{1}{2} \partial^{2} F_{5}(s, z) y_{1}(s, z)^{2} \\
& +\frac{1}{6} \partial F_{4}(s, z) y_{3}(s, z)+\frac{1}{2} \partial^{2} F_{4}(s, z) y_{1}(s, z) y_{2}(s, z) \\
& +\frac{1}{6} \partial^{3} F_{4}(s, z) y_{1}(s, z)^{3}+\frac{1}{24} \partial F_{3}(s, z) y_{4}(s, z) \\
& +\frac{1}{6} \partial^{2} F_{3}(s, z) y_{1}(s, z) y_{3}(s, z)+\frac{1}{4} \partial^{3} F_{3}(s, z) y_{1}(s, z)^{2} y_{2}(s, z) \\
& +\frac{1}{24} \partial^{4} F_{3}(s, z) y_{1}(s, z)^{4}+\frac{1}{8} \partial^{2} F_{3}(s, z) y_{2}(s, z)^{2} \\
& +\frac{1}{120} \partial F_{2}(s, z) y_{5}(s, z)+\frac{1}{24} \partial^{2} F_{2}(s, z) y_{1}(s, z) y_{4}(s, z) \\
& +\frac{1}{8} \partial^{3} F_{2}(s, z) y_{1}(s, z) y_{2}(s, z)^{2}+\frac{1}{12} \partial^{3} F_{2}(s, z) y_{1}(s, z)^{2} y_{3}(s, z) \\
& +\frac{1}{12} \partial^{4} F_{2}(s, z) y_{1}(s, z)^{3} y_{2}(s, z)+\frac{1}{120} \partial^{5} F_{2}(s, z) y_{1}(s, z)^{5} \\
& +\frac{1}{12} \partial^{2} F_{2}(s, z) y_{2}(s, z) y_{3}(s, z)+\frac{1}{720} \partial F_{1}(s, z) y_{6}(s, z) \\
& +\frac{1}{120} \partial^{2} F_{1}(s, z) y_{1}(s, z) y_{5}(s, z)+\frac{1}{12} \partial^{3} F_{1}(s, z) y_{1}(s, z) y_{2}(s, z) y_{3}(s, z) \\
& +\frac{1}{48} \partial^{3} F_{1}(s, z) y_{1}(s, z)^{2} y_{4}(s, z)+\frac{1}{16} \partial^{4} F_{1}(s, z) y_{1}(s, z)^{2} y_{2}(s, z)^{2} \\
& +\frac{1}{36} \partial^{4} F_{1}(s, z) y_{1}(s, z)^{3} y_{3}(s, z)+\frac{1}{48} \partial^{5} F_{1}(s, z) y_{1}(s, z)^{4} y_{2}(s, z) \\
& +\frac{1}{120} \partial^{6} F_{1}(s, z) y_{1}(s, z)^{6}+\frac{1}{48} \partial^{2} F_{1}(s, z) y_{2}(s, z) y_{4}(s, z) \\
& \left.+\frac{1}{48} \partial^{3} F_{1}(s, z) y_{2}(s, z)^{3}+\frac{1}{72} \partial^{2} F_{1}(s, z) y_{3}(s, z)^{2}\right) d s .
\end{aligned}
$$

If we denote the solution of the differential system (6) with $x(t, z, \varepsilon)$ such that $x(0, z, \varepsilon)=z$, then we have

$$
x(T, z, \varepsilon)-z=\sum_{i=1}^{7} \varepsilon f_{i}(z)+\ldots
$$

Therefore by using the Implicit Function Theorem we conclude that if $f_{1}(z)=f_{2}(z)=\ldots=f_{k-1}(z) \equiv 0$ and $f_{k}(z) \not \equiv 0$, then the simple zeros 
of $f_{k}(z)$ provide limit cycles of the differential system (6). For more details see Theorem A of [27].

\section{Proof of Theorem 2}

Before proving Theorem 2 we prove Lemma 1.

Proof of Lemma 1. First we translate the center $\left(\left(1-\sqrt{1+4 r^{2}}\right) / 2,0\right)$ of the differential system (1) to the origin of coordinates and we obtain the differential system

$$
\dot{x}=y, \quad \dot{y}=-\sqrt{1+4 r^{2}} x+x^{2}+y^{2} .
$$

Doing the linear change $(x, y)=\left(v / \sqrt[4]{\left(1+4 r^{2}\right)}, u\right)$ we write the linear part of the differential system (7) into its canonical real Jordan normal form, and system (7) becomes

$$
\begin{aligned}
& \dot{u}=-\sqrt[4]{\left(1+4 r^{2}\right)} v+u^{2}+\frac{v^{2}}{\sqrt{1+4 r^{2}}}, \\
& \dot{v}=\sqrt[4]{\left(1+4 r^{2}\right)} u
\end{aligned}
$$

Performing the transformation $u=\alpha X, v=\beta Y$ with

$$
\alpha=-\beta=\sqrt[4]{\left(1+4 r^{2}\right)^{3}}\left(\frac{\sqrt{1+4 r^{2}}-1}{r^{2}}\right),
$$

and changing $(X, Y)$ by $(x, y)$ the differential system (8) becomes system (3) with $A=\frac{1-\sqrt{1+4 r^{2}}}{r^{2}}$. Moreover system (3) is into the form (2) of reversible quadratic centers taking $a=b=1+\frac{A}{2}$.

Proof of Theorem 1. The aim of this section is to study the limit cycles of the quadratic system (5) when $\varepsilon \neq 0$ is sufficiently small by using the averaging theory of six order.

We see that system (5) has a center at the origin and the linear part of the system is in its canonical real Jordan normal form when $\varepsilon=0$. We consider the change to polar coordinates $x=r \cos \theta, y=r \sin \theta$ and we get the differential system (5) in polar coordinates $(\dot{r}, \dot{\theta})$.

After rescaling the variable $r$ as $r=\varepsilon R$ and taking the angle $\theta$ as the new independent variable, we obtain the differential equation

$$
\frac{d R}{d \theta}=\sum_{i=1}^{7} \varepsilon^{i} F_{i}(\theta, R)+O\left(\varepsilon^{7}\right)
$$


Now we can apply the averaging theory described in section 2 to this differential system, because system (9) is equivalent to system (6) taking $x=R, t=\theta$ and $T=2 \pi$.

For the differential equation (9) we have that $F_{i}=F_{i}(\theta, R)$ for $i=$ $1,2,3,4$ are

$$
\begin{aligned}
F_{1}= & -\lambda_{11} R+-\cos \theta((A+2) \cos (2 \theta)+2) R^{2} \\
F_{2}= & -\lambda_{12} R+\sin \theta\left(\cos (2 \theta)\left((A+2) \lambda_{11}-2 \lambda_{51}\right)+2 \lambda_{11}+\lambda_{31}-\lambda_{51}\right) R^{2} \\
& +\frac{1}{2} \sin (2 \theta)((A+2) \cos (2 \theta)+2)^{2} R^{3} \\
F_{3}= & -\lambda_{13} R+\left(\sin \theta\left(\cos (2 \theta)\left((A+2) \lambda_{12}-2 \lambda_{52}\right)+2 \lambda_{12}+\lambda_{32}-\lambda_{52}\right)\right. \\
& -\lambda_{11}\left(\lambda_{31} \cos \theta+\lambda_{51} \cos (3 \theta)\right) R^{2}+\frac{1}{4}((A+2) \cos (2 \theta)+2) \\
& \left(\cos (4 \theta)\left((A+2) \lambda_{11}-4 \lambda_{51}\right)-2 A \lambda_{11} \cos (2 \theta)\right. \\
& \left.+(A-2) \lambda_{11}-4 \lambda_{31}\right) R^{3}-\sin ^{2} \theta \cos \theta((A+2) \cos (2 \theta)+2)^{3} R^{4}, \\
F_{4}= & -\lambda_{14} R+\left(\sin \theta\left(\cos (2 \theta)\left((A+2) \lambda_{13}-2 \lambda_{53}\right)+2 \lambda_{13}+\lambda_{33}-\lambda_{53}\right)\right. \\
& \left.+\cos \theta\left(-\lambda_{11} \lambda_{32}-\lambda_{12} \lambda_{31}\right)-\cos (3 \theta)\left(\lambda_{11} \lambda_{52}+\lambda_{12} \lambda_{51}\right)\right) R^{2} \\
& +\frac{1}{8}\left(-2 \cos (4 \theta)\left(\left(A^{2}-4\right) \lambda_{12}+8 \lambda_{52}\right)-2\left(\left(A^{2}+4\right) \lambda_{12}+8 \lambda_{32}\right)\right. \\
& +4 \sin (2 \theta)\left((A-2) \lambda_{11} \lambda_{51}+4 \lambda_{11} \lambda_{31}+\lambda_{31}\left(\lambda_{31}-2 \lambda_{51}\right)\right) \\
& +4 \lambda_{11} \sin (4 \theta)\left((A+2) \lambda_{31}-(A-2) \lambda_{51}\right)+4 \lambda_{51} \sin (6 \theta) \\
& \left((A+2) \lambda_{11}-\lambda_{51}\right)+\cos (2 \theta)\left((A-2)(3 A+2) \lambda_{12}\right. \\
& \left.\left.-4(A+2)\left(2 \lambda_{32}+\lambda_{52}\right)\right)+(A+2) \cos (6 \theta)\left((A+2) \lambda_{12}-4 \lambda_{52}\right)\right) R^{3} \\
& +\frac{1}{4} \sin \theta((A+2) \cos (2 \theta)+2)^{2}\left(2 \cos (2 \theta)\left(A \lambda_{11}+\lambda_{31}+\lambda_{51}\right)\right. \\
& \left.-\cos (4 \theta)\left((A+2) \lambda_{11}-6 \lambda_{51}\right)-A \lambda_{11}+2 \lambda_{11}+6 \lambda_{31}\right) R^{4} \\
& +\sin { }^{3} \theta \cos \theta((A+2) \cos (2 \theta)+2)^{4} R^{5} .
\end{aligned}
$$

We do not provide the functions $F_{i}(\theta, R)$ for $i=5,6,7$ because their expressions are huge, and they are easy to compute with the help of an algebraic manipulator as mathematica or mapple.

From the formulas of section 2 we can compute again by using an algebraic manipulator as mathematica or mapple the average functions. Thus the average function of first order is

$$
f_{1}(R)=-\lambda_{11} R \text {. }
$$

It is clear that $f_{1}(R)=0$ has no positive roots. Therefore we get no information about the bifurcated limit cycles of system (5) from the 
average function of first order. By setting $\lambda_{11}=0$, we have $f_{1}(R) \equiv 0$. So computing the average function of second order we obtain

$$
f_{2}(R)=-\lambda_{12} R
$$

Again $f_{2}(R)$ does not provide any information about the limit cycles. Taking $\lambda_{12}=0$ we compute the third order average function

$$
f_{3}(R)=-\lambda_{13} R-2 \lambda_{31} R^{3} .
$$

Clearly equation $f_{3}(R)=0$ has at most one simple positive root, showing that the third average function can provide one limit cycle for the differential equation (9).

If $\lambda_{13}=\lambda_{31}=0$, then $f_{3}(R) \equiv 0$ and we compute the fourth order average function

$$
f_{4}(R)=-\lambda_{14} R-2 \lambda_{32} R^{3} .
$$

Therefore as in the case of the third average function the fourth one can provide at most one limit cycle for the differential equation (9).

By setting $\lambda_{14}=\lambda_{32}=0$ we compute

$$
f_{5}(R)=-\lambda_{15} R-2 \lambda_{33} R^{3}-\frac{1}{3}(A-6)(A+4) \lambda_{51} R^{5} .
$$

It follows immediately that equation $f_{5}(R)=0$ can have at most two simple positive roots if $A \notin\{-4,6\}$, and consequently the fifth average function can provide at most two limit cycles for the differential equation (9) if $A \notin\{-4,6\}$. When $A \in\{-4,6\}$ as for the third average function the fifth average function can provide at most one limit cycle for the differential equation (9). Now we must distinguish three cases.

Case 1: $\lambda_{15}=\lambda_{33}=\lambda_{51}=0$. Then we compute the sixth order averaging function and we obtain

$$
f_{6}(R)=-\lambda_{16} R-2 \lambda_{34} R^{3}-\frac{1}{3}(A-6)(A+4) \lambda_{52} R^{5} .
$$

So the same conclusions than for the fifth average function, and we must consider three subcases.

Subcase 1.1: $\lambda_{16}=\lambda_{34}=\lambda_{52}=0$ and we obtain the seventh order averaging function

$$
f_{7}(R)=-\lambda_{17} R-2 \lambda_{35} R^{3}-\frac{1}{3}(A-6)(A+4) \lambda_{53} R^{5} .
$$

Again the same conclusions than for the fifth average function.

Subcase 1.2: $\lambda_{16}=\lambda_{34}=0, A=-4$. Then the seventh order averaging function is

$$
f_{7}(R)=-\lambda_{17} R-2 \lambda_{35} R^{3}
$$


So equation $f_{7}(R)=0$ can have at most one simple positive root, and consequently the seventh average function can provide at most one limit cycle for the differential equation (9).

Subcase 1.3: $\lambda_{16}=\lambda_{34}=0, A=6$. Therefore the seventh order averaging function is (10). So the same conclusion than in the subcase 1.2 .

Case 2: $\lambda_{15}=\lambda_{33}=0, A=-4$ and we obtain the sixth order averaging function

$$
f_{6}(R)=-\lambda_{16} R-2 \lambda_{34} R^{3} .
$$

Therefore in this case we can obtain at most one limit cycle for the differential equation (9). Taking $\lambda_{16}=\lambda_{34}=0$ we get that the seventh order averaging function is

$$
f_{7}(R)=-\lambda_{17} R-2 \lambda_{35} R^{3}+\frac{11011}{1296} \lambda_{51} R^{7} .
$$

Hence equation $f_{7}(R)=0$ can have at most two simple positive roots, and consequently the seventh average function can provide at most two limit cycles for the differential equation (9). In short, when $A=-4$ the two limit cycles appear in order seven and not in order five as it is the case for $A \in \mathbb{R} \backslash\{-4,6\}$.

Case 3: $\lambda_{15}=\lambda_{33}=0, A=6$ and we obtain the sixth order average function is (11). So at most one limit cycle at order sixth. Taking $\lambda_{16}=\lambda_{34}=0$ we get that the seventh order averaging function is

$$
f_{7}(R)=-\lambda_{17} R-2 \lambda_{35} R^{3}-\frac{193594}{81} \lambda_{51} R^{7} .
$$

Again as in the case $A=-4$ for $A=6$ the two limit cycles do not appear until order seven.

This completes the proof of Theorem 2.

\section{ACKNOWLEDGEMENTS}

This work is supported by the Ministerio de Economì, Industria y competitividad, Agencia Estatal de Investigación grant MTM201677278-P (FEDER), the Agència de Gestió d'Ajusts Universitaris i de Recerca grant 2017 SGR 1617, and the European project DynamicsH2020-MSCA-RISE-2017-777911. The second and third authors are supported by Isfahan University of Technology (IUT). 


\section{REFERENCES}

[1] A. Atabaigi, N. Nyamoradi and H. Zangeneh, The number of limit cycles of a quintic polynomial system with a center, Nonlinear Anal. 7 (2009), 30083017.

[2] R. Benterki And J. Llibre, Centers and limit cycles of polynomial differential systems of degree 4 via averaging theory, J. Comput. Appl. Math. 407 (2013), 16-22.

[3] T. Blows And L. Perko, Bifurcation of limit cycles from centers and separatrix cycles of planar analytic systems, SIAM Rev. 36 (1994), 341-376.

[4] G. Chen, C. Li, C. LiU And J. Llibre, The cyclicity of period annuli of some classes of reversible quadratic systems, Disc. Contin. Dyn. Sys. 16 (2006), $157-177$.

[5] F. Chen, C. Li, J. Llibre And Z. Zhang, A unified proof on the weak Hilbert 16th problem for $n=2$, J. Differential Equations 221 (2006), 309-342

[6] C. Christopher And C. Li, Limit cycles of differential equations, Advanced Courses in Mathematics. CRM Barcelona. Birkhuser Verlag, Basel, 2007.

[7] B. Coll, J. Llibre And R. Prohens, Limit cycles bifurcating from a perturbed quartic center, Chaos Solitons Fractals 44 (2011), 317-334.

[8] F. Dumortier And C. LI, Perturbations from an elliptic Hamiltonian of degree four: (IV) figure eight loop, J. Diff. Eqs. 188 (2003), 512-554.

[9] F. Dumortier, J. Llibre And J.C. Artés, Qualitative Theory of Planar Differential Systems, Springer Verlag, New York, 2006.

[10] L. Gavrilov And E. Horozov, Limit cycles of perturbations of quadratic Hamiltonian vector fields, J. Math. Pures et appl. 72 (1993), 213-238.

[11] H. Giacomini, J. Llibre And M. Viano, On the shape of limit cycles that bifurcate from Hamiltonian centers, Nonlinear Anal. 41 (2000), 523-537.

[12] M. HAn, Asymptotic expansions of Melnikov functions and limit cycle bifurcations, Int. J. Bifur. Chaos 22(12) (2012) 1250296 (30 pp).

[13] M. HAN, J. YANG AND P. YU, Hopf bifurcations for near-Hamiltonian systems, Int. J. Bifur. Chaos 19 (2009), 4117-4130.

[14] M. HAN AND P. YU, Normal forms, Melnikov functions and bifurcations of limit cycles, Applied Mathematical Sciences, vol. 181, Springer, London, 2012.

[15] E. Horozov AND I.D. Iliev, On the number of limit cycles in perturbations of quadratic Hamiltonian systems, Proc. London Math. Soc. 69 (1994), 198-224.

[16] I.D. ILIEv, High-order Melnikov functions for degenerate cubic Hamiltonian, Adv. Diff. Eqns. 1 (1996), 689-708.

[17] I.D. Iliev, Perturbations of quadratic centers, Bull. Sci. Math. 122 (1998), 107-161.

[18] I.D. ILIEv, C. Li AND J. Yu, Bifurcation of limit cycles from quadratic nonHamiltonian systems with two centers and two unbounded heteroclinic loops, Nonlinearity 18 (2005), 305-330.

[19] C. Li AND J. Llibre, A unified study on the cyclicity of period annulus or reversible quadratic Hamiltonian systems, J. Dynamics and Differential Equations 16 (2004), 271-295.

[20] C. Li, J. Llibre AND Z. ZHAng, Weak focus, limit cycles and bifurcations for bounded quadratic systems, J. Diff. Eqns. 115 (1995), 193-223.

[21] C. Li AND Z. ZhAng, Remarks on 16th weak Hilbert problem for $n=2$, Nonlinearity 15 (2002), 1975-1992. 
[22] J. LI, Limit cycles bifurcated from a reversible quadratic center, Qual. Theory Dyn. Syst. 6 (2002), 205-215.

[23] C. LiU, Limit cycles bifurcated from some reversible quadratic centers with a non-algebraic first integral, Nonlinearity 25 (2012), 1653-1660.

[24] J. Llibre, R. Moeckel and C. Simó, Central Configurations, Periodic Orbits, and Hamiltonian Systems, Advanced Courses in Mathematics, CRM Barcelona, Birkhuser, Springer, Basel, 2015.

[25] J. Llibre, M. Mousavi And A. Nabavi, New family of centers of planar polynomial differential systems of arbitrary even degree, J. Dyn Control Syst. Submitted.

[26] S. Smale, Mathematical problems for the next century, Math. Intell. 20 (1998), $7-15$.

[27] J. Llibre, D.D. Novaes And M.A. TeixeIRA, Higher order averaging theory for finding periodic solutions via Brouwer degree, Nonlinearity 27 (2014), 563583.

[28] G. XiAng And M. Han, Global bifurcation of limit cycles in a family of polynomial systems, J. Math. Anal. Appl. 295 (2004), 633-644.

${ }^{1}$ Departament de Matemàtiques, Universitat Autònoma de Barcelona, 08193 Bellaterra, Barcelona, Catalonia, Spain

E-mail address: jllibre@mat.uab.cat

2 Department of Mathematical Sciences, Isfahan University of TechNOLOGY, ISFAHAN, IRAN, 84156-83111.

E-mail address: a.nabavi@math.iut.ac.ir

E-mail address: marzieh.mousavi@math.iut.ac.ir 